\title{
A polarimetric view of the open cluster Lyngå 6 and the Cepheid TW Normæ
}

\author{
A. M. Orsatti ${ }^{1,2,4,5}$, E. I. Vega ${ }^{1,3,4,5}$, and H. G. Marraco ${ }^{1,4}$ \\ 1 Facultad de Ciencias Astronómicas y Geofísicas (Universidad Nacional de La Plata), Paseo del Bosque s/n, \\ 1900, La Plata, Argentina \\ 2 Instituto de Astrofísica de La Plata (IALP, CONICET), Paseo del Bosque s/n, 1900, La Plata, Argentina \\ 3 Instituto de Astronomía y Física del Espacio, C. C. 67 Suc. 28, 1428 Buenos Aires, Argentina \\ ${ }^{4}$ Member of the Carrera del Investigador Científico of CONICET \\ 5 Visiting Astronomer, Complejo Astronómico El Leoncito, operated under agreement between the Consejo \\ Nacional de Investigaciones Científicas y Técnicas de la República Argentina and the Universities of La Plata, \\ Córdoba, and San Juan
}

Received 19 July 2001 / Accepted 16 August 2001

\begin{abstract}
We present $U B V R I$ polarimetric observations of stars belonging to the small open cluster Lyngå 6 and of TW Nor, a Cepheid which can be seen near the center of the grouping. The wavelength of maximum polarization is computed and then analyzed in the context of its relation to the optical properties and characteristic particle size distribution of the grains responsible for the polarization. The amount and direction of linear polarization is also computed. Results indicate a mean polarization efficiency close to the value found for the interstellar medium. Two out of seven members of Lyngå 6 present indications of intrinsic polarization in their measures. We conclude that part of the polarization detected in the light from members of the cluster comes from a dust cloud located in the Local arm, at a distance of about $1 \mathrm{kpc}$. In general, polarization measurements do not support the membership of TW Nor to Lyngå 6 .
\end{abstract}

Key words. ISM: dust, extinction - open clusters and associations: individual (Lyngå 6) - stars: individual (TW Nor) - Cepheids

\section{Introduction}

Lyngå 6 (C1512-581) is a small group of young stars, $4^{\prime}$ in diameter, which is located in the Sagittarius spiral arm at $l=3300^{\circ}$ and $b=+0.30$. It was first detected and listed as a suspected cluster by Lyngå (1964). Madore (1975) and Moffat \& Vogt (1975), almost simultaneously, both made the first photoelectric observations of the possible members but they reached opposite conclusions: while the first author supported the idea of it lying in the presence of a real cluster, in the other investigation doubts were raised.

New investigations by van den Bergh \& Harris (1976), Thé (1977), Lyngå (1977), Schmidt (1983) and Walker (1985) confirmed that it was a real grouping. True distance moduli from these investigations ranged rather widely: 11.7 (Madore 1975), $10.8 \pm 0.6$ (van den Bergh \& Harris 1976), 10.8 (Lyngå 1977); and between 10.6 and

Send offprint requests to: H. G. Marraco,

e-mail: hmarraco@conae.gov.ar
10.9 mag (Schmidt 1983). The large uncertainties present in some of these determinations may be attributed to different origins: the handling of the problem of evolution, the presence of unresolved binaries, the ZAMS fitting to the nearly vertical MS, etc. They were removed with the work of Walker (1985), who performed CCD photometry in the $(B, V, I)$ bands down to $V=19$ mag. This led to an accurate fitting of the ZAMS from which a value for the true distance modulus of $11.15 \pm 0.3 \mathrm{mag}$ was found, with a mean excess $E_{B-V}$ of $1.34 \pm 0.01$ mag. It is thought to be the safest fit to the ZAMS, until individual reddening measurements are available.

The reason for such an interest in Lyngå 6 is TW Nor, a Cepheid which can be seen near the center of the grouping. Cepheids in Galactic clusters provide the most straightforward mean of performing the calibration of the PeriodLuminosity-Color relation (P-L-C); and TW Nor was part of this selected group. Moreover, it was a particularly interesting object as it is the longest period Cepheid 
believed to be a member of an open cluster, with $P=$ 10.7845 (Caldwell \& Coulson 1987).

The membership of TW Nor in Lyngå 6 has been a matter of discussion for many years. While some investigators clearly agree with its location in the open cluster, others find that some incongruences exist between the observational parameters for the cluster and those for the Cepheid.

The polarimetric techniques can be a very useful tool in providing clues for membership problems. With polarimetry, we can get important information on the characteristics $\left(P_{\max }, \lambda_{\max }\right.$, magnetic field direction, etc.) of the dust located in front of an object, or the dust parameters associated with a group of stars. In this paper we provide a polarimetric view of the problem involving Lyngå 6 and TW Nor. To do so, we propose first to investigate the properties of the dust in front of the open cluster as a whole and then to check if they are coincident with the ones for the dust located just in front of the Cepheid.

\section{Observations}

We have selected for polarimetric observation 7 suspected members of Lyngå 6 (stars $2,4,6,7,8,9,10)$ and 8 non-members $(1,13,14,16,17,18,19,20)$ according to Lyngå (1977), and also TW Nor.

Observations in the $U B V R_{\mathrm{KC}} I_{\mathrm{KC}}$ bands (KC: KronCousins, $\lambda_{U_{\text {eff }}}=0.36 \mu \mathrm{m}, F W H M=0.05 \mu \mathrm{m} ; \lambda_{B_{\text {eff }}}=$ $0.44 \mu \mathrm{m}, F W H M=0.06 \mu \mathrm{m} ; \lambda_{V_{\text {eff }}}=0.53 \mu \mathrm{m}$, $F W H M=0.06 \mu \mathrm{m} ; \lambda_{R_{\text {eff }}}=0.69 \mu \mathrm{m}, F W H M=$ $\left.0.18 \mu \mathrm{m} ; \lambda_{I_{\mathrm{eff}}}=0.83 \mu \mathrm{m}, F W H M=0.15 \mu \mathrm{m}\right)$ were carried out using the five-channel photopolarimeter of the Torino Astronomical Observatory attached to the $2.15 \mathrm{~m}$ telescope at the Complejo Astronómico El Leoncito (San Juan, Argentina). They were performed on 2 nights (March 22-23) in 1998 and 1 night (June 30) in 2000. Standard stars for null polarization and for the zero point of the polarization position angle were taken from Clocchiati \& Marraco (1988). Table 1 lists the observed stars in the direction of Lyngå 6 , the percentage polarization $\left(P_{\lambda}\right)$, the position angle of the electric vector $\left(\theta_{\lambda}\right)$ in the equatorial coordinate system and their respective mean errors for each filter. We indicate also the number of $60 \mathrm{~s}$ independent integrations with each filter. Star identifications are taken from the work of Lyngå (1977), and non-members according to that work are indicated in this table and also in Table 2 below.

\section{Results}

By observing the amount of interstellar polarization in several bandpasses, the wavelength at which maximum polarization $\left(P_{\max }\right)$ occurs can be computed. This wavelength $\lambda_{\max }$ is a function of the optical properties and characteristic particle size distribution of the aligned grains (McMillan 1978; Wilking et al. 1980). The maximum polarization at which $P_{\max }$ occurs has been
Table 1. UBVRI Lyngå 6 polarizations.

\begin{tabular}{|c|c|c|c|c|c|c|}
\hline $\operatorname{Star}^{a}$ & filter & $\begin{array}{c}P_{\lambda} \\
\%\end{array}$ & $\epsilon_{p}$ & $\begin{array}{c}\theta_{\lambda} \\
\circ\end{array}$ & $\overline{\overline{\epsilon_{\theta}}}$ & $\overline{n^{b}}$ \\
\hline \multirow[t]{5}{*}{$1^{*}$} & $\bar{U}$ & 1.39 & 0.12 & 33.2 & 2.4 & 6 \\
\hline & $B$ & 2.01 & 0.08 & 30.2 & 1.2 & \\
\hline & $V$ & 2.06 & 0.07 & 31.0 & 1.0 & \\
\hline & $R$ & 2.06 & 0.06 & 29.3 & 0.8 & \\
\hline & $I$ & 1.76 & 0.19 & 31.3 & 3.0 & \\
\hline \multirow[t]{5}{*}{2} & $U$ & - & - & - & - & 4 \\
\hline & $B$ & 6.04 & 0.43 & 42.0 & 2.3 & \\
\hline & $V$ & 6.28 & 0.79 & 39.2 & 1.8 & \\
\hline & $R$ & 5.80 & 0.35 & 38.7 & 2.0 & \\
\hline & $I$ & 5.93 & 0.48 & 35.4 & 3.7 & \\
\hline \multirow[t]{5}{*}{4} & $U$ & - & - & - & - & 4 \\
\hline & $B$ & 5.63 & 0.30 & 38.6 & 1.5 & \\
\hline & V & 5.76 & 0.06 & 38.5 & 0.3 & \\
\hline & $R$ & 6.09 & 0.05 & 40.0 & 0.2 & \\
\hline & $I$ & 5.55 & 0.10 & 40.7 & 0.5 & \\
\hline \multirow[t]{5}{*}{6} & $U$ & - & - & - & - & 12 \\
\hline & $B$ & 5.11 & 1.11 & 34.7 & 6.1 & \\
\hline & $V$ & 4.98 & 0.42 & 40.7 & 2.4 & \\
\hline & $R$ & 5.36 & 0.34 & 38.1 & 1.8 & \\
\hline & $I$ & 4.50 & 0.48 & 39.4 & 3.0 & \\
\hline \multirow[t]{5}{*}{7} & $U$ & 4.30 & 0.60 & 38.1 & 4.0 & 11 \\
\hline & $B$ & 4.91 & 0.45 & 35.9 & 2.6 & \\
\hline & $V$ & 5.22 & 0.12 & 40.8 & 0.7 & \\
\hline & $R$ & 5.51 & 0.14 & 40.5 & 0.7 & \\
\hline & $I$ & 4.83 & 0.26 & 43.0 & 1.6 & \\
\hline \multirow[t]{5}{*}{8} & $U$ & - & - & - & - & 4 \\
\hline & $B$ & 4.55 & 0.46 & 45.3 & 2.9 & \\
\hline & $V$ & 4.89 & 0.11 & 39.5 & 0.7 & \\
\hline & $R$ & 4.94 & 0.11 & 38.8 & 0.6 & \\
\hline & $I$ & 5.11 & 0.18 & 38.6 & 1.0 & \\
\hline \multirow[t]{5}{*}{9} & $U$ & - & - & - & - & 2 \\
\hline & $B$ & 5.79 & 0.91 & 44.0 & 2.2 & \\
\hline & $V$ & 6.70 & 0.69 & 38.3 & 1.5 & \\
\hline & $R$ & 5.80 & 0.44 & 39.5 & 1.1 & \\
\hline & $I$ & 5.84 & 0.39 & 36.3 & 0.9 & \\
\hline \multirow[t]{5}{*}{10} & $U$ & 5.57 & 1.02 & 43.6 & 5.2 & 8 \\
\hline & $B$ & 6.31 & 0.37 & 41.0 & 1.7 & \\
\hline & $V$ & 5.38 & 0.19 & 38.4 & 1.0 & \\
\hline & $R$ & 5.74 & 0.11 & 39.2 & 0.6 & \\
\hline & $I$ & 5.21 & 0.28 & 38.9 & 1.5 & \\
\hline
\end{tabular}

calculated by fitting the observed polarization in the $U B V R I$ bandpasses to the standard Serkowski's polarization law,

$P_{\lambda} / P_{\max }=\exp \left[\begin{array}{lll}-k & \ln ^{2}\left(\lambda_{\max } / \lambda\right)\end{array}\right]$

and adopting the parameter $k=1.15$ (Serkowski 1973). If the polarization is well represented by this relation 
Table 1. continued.

\begin{tabular}{|c|c|c|c|c|c|c|}
\hline $\operatorname{Star}^{a}$ & filter & $\begin{array}{c}P_{\lambda} \\
\%\end{array}$ & $\bar{\epsilon}$ & $\begin{array}{c}\theta_{\lambda} \\
\circ\end{array}$ & $\bar{\epsilon} \epsilon_{\theta}$ & $\overline{n^{b}}$ \\
\hline \multirow[t]{5}{*}{13} & $U$ & 4.64 & 0.41 & 49.8 & 2.5 & 4 \\
\hline & $B$ & 5.70 & 0.29 & 35.6 & 1.4 & \\
\hline & $V$ & 5.92 & 0.28 & 37.0 & 1.3 & \\
\hline & $R$ & 5.87 & 0.23 & 38.1 & 1.1 & \\
\hline & $I$ & 5.07 & 0.21 & 36.9 & 1.2 & \\
\hline \multirow[t]{5}{*}{$14^{*}$} & $U$ & - & - & - & - & 4 \\
\hline & $B$ & 2.27 & 0.30 & 41.6 & 1.8 & \\
\hline & $V$ & 2.28 & 0.17 & 34.3 & 1.1 & \\
\hline & $R$ & 2.23 & 0.14 & 27.6 & 0.9 & \\
\hline & $I$ & 2.02 & 0.10 & 27.1 & 0.7 & \\
\hline \multirow[t]{5}{*}{$16^{*}$} & $U$ & 2.04 & 0.11 & 36.1 & 1.6 & 2 \\
\hline & $B$ & 2.71 & 0.11 & 32.9 & 1.2 & \\
\hline & $V$ & 2.71 & 0.08 & 37.9 & 0.8 & \\
\hline & $R$ & 2.68 & 0.12 & 33.4 & 1.3 & \\
\hline & $I$ & 2.63 & 0.18 & 38.1 & 2.0 & \\
\hline \multirow[t]{5}{*}{$17^{*}$} & $U$ & 4.29 & 0.22 & 45.8 & 1.5 & 2 \\
\hline & $B$ & 4.80 & 0.18 & 43.8 & 1.0 & \\
\hline & $V$ & 4.43 & 0.09 & 43.1 & 0.6 & \\
\hline & $R$ & 4.55 & 0.08 & 42.6 & 0.5 & \\
\hline & $I$ & 4.40 & 0.17 & 42.9 & 1.1 & \\
\hline \multirow[t]{5}{*}{$18^{*}$} & $U$ & 5.35 & 0.23 & 43.4 & 1.2 & 2 \\
\hline & $B$ & 5.16 & 0.17 & 41.4 & 0.9 & \\
\hline & $V$ & 5.07 & 0.14 & 40.5 & 0.8 & \\
\hline & $R$ & 5.53 & 0.11 & 40.8 & 0.6 & \\
\hline & $I$ & 5.03 & 0.17 & 40.6 & 1.0 & \\
\hline \multirow[t]{5}{*}{$19^{*}$} & $U$ & 1.46 & 0.29 & 33.2 & 5.5 & 10 \\
\hline & $B$ & 2.12 & 0.06 & 32.4 & 0.8 & \\
\hline & $V$ & 2.13 & 0.12 & 30.4 & 1.6 & \\
\hline & $R$ & 2.20 & 0.09 & 30.3 & 1.1 & \\
\hline & $I$ & 2.08 & 0.15 & 30.8 & 2.1 & \\
\hline \multirow[t]{5}{*}{$20^{*}$} & $U$ & - & - & - & - & 4 \\
\hline & $B$ & 2.06 & 0.34 & 24.5 & 4.7 & \\
\hline & $V$ & 2.18 & 0.11 & 30.3 & 1.4 & \\
\hline & $R$ & 2.29 & 0.10 & 28.5 & 1.3 & \\
\hline & $I$ & 2.10 & 0.14 & 29.6 & 1.9 & \\
\hline \multirow[t]{5}{*}{ TW Nor } & $U$ & - & - & - & - & 2 \\
\hline & $B$ & 7.14 & 0.61 & 44.3 & 1.10 & \\
\hline & $V$ & 7.23 & 0.13 & 41.0 & 0.25 & \\
\hline & $R$ & 7.31 & 0.08 & 34.4 & 0.15 & \\
\hline & $I$ & 6.52 & 0.05 & 36.0 & 0.11 & \\
\hline
\end{tabular}

${ }^{a}$ Lyngå (1977).

${ }^{b}$ Number of $60 \mathrm{~s}$ integrations.

* Stands for non-member.

(meaning that the measured polarization is of interstellar origin), $\sigma_{1}$ (the unit weight error of the fit) should not be higher than 1.5, because of the weighting scheme; a higher value could indicate the presence of intrinsic polarization.
Table 2. Polarization results of stars in Lyngå 6 .

\begin{tabular}{lcccccc}
\hline \hline Star $^{a}$ & $\begin{array}{c}P_{\max } \\
\%\end{array}$ & $\epsilon_{p}$ & $\sigma_{1}^{b}$ & $\begin{array}{c}\lambda_{\max } \\
\mu \mathrm{m}\end{array}$ & $\begin{array}{c}\epsilon_{\lambda} \\
E_{B-V} \\
\mathrm{mag}\end{array}$ \\
\hline $1^{*}$ & 2.09 & 0.06 & 1.28 & 0.60 & 0.03 & 0.20 \\
2 & 6.36 & 0.29 & 1.12 & 0.59 & 0.05 & 1.36 \\
4 & 6.06 & 0.06 & 1.46 & 0.64 & 0.02 & 1.36 \\
6 & 5.26 & 0.15 & 0.57 & 0.59 & 0.04 & 1.33 \\
7 & 5.50 & 0.06 & 0.95 & 0.61 & 0.02 & 1.36 \\
8 & 5.13 & 0.14 & 1.73 & 0.66 & 0.04 & 1.37 \\
9 & 6.33 & 0.31 & 0.99 & 0.61 & 0.05 & 1.38 \\
10 & 5.85 & 0.21 & 2.07 & 0.57 & 0.05 & 1.31 \\
13 & 6.01 & 0.04 & 0.30 & 0.57 & 0.01 & 1.11 \\
$14^{*}$ & 2.33 & 0.04 & 0.44 & 0.58 & 0.02 & 1.05 \\
$16^{*}$ & 2.81 & 0.08 & 1.28 & 0.60 & 0.03 & 0.33 \\
$17^{*}$ & 4.73 & 0.17 & 2.93 & 0.56 & 0.04 & 0.74 \\
$18^{*}$ & 5.65 & 0.25 & 3.32 & 0.56 & 0.04 & 0.75 \\
$19^{*}$ & 2.28 & 0.06 & 1.03 & 0.59 & 0.03 & 0.27 \\
$20^{*}$ & 2.29 & 0.02 & 0.26 & 0.64 & 0.01 & - \\
TW Nor & 7.39 & 0.05 & 0.62 & 0.60 & 0.01 & 1.34 \\
\hline
\end{tabular}

${ }^{a}$ Lyngå (1977).

${ }^{b} \sigma_{1}{ }^{2}=\sum\left(r_{\lambda} / \epsilon_{p_{\lambda}}\right)^{2} /(m-2)$; where $m$ is the number of colors and $r_{\lambda}=P_{\lambda}-P_{\max } \exp \left(-K \ln ^{2}\left(\lambda_{\max } / \lambda\right)\right)$.

* Stands for non-member.

Table 2 lists the $P_{\max }$ and $\lambda_{\max }$ values for the observed stars. The mathematical expression used to determine the individual $\sigma_{1}$ values can be found there as a footnote. The last column in this table lists the color excesses $E_{B-V}$ for each star. There is practically no information in the literature about the spectral types for the observed stars. No. 16 is identified as HD 143738, a B4 V star (Knude 1992); and from objective prism plates, Lyngå (1977) proposes that Nos. 1 and 19 are late B stars on the main sequence, No. 17 is an OB object and No. 4 could be a G5 III star. Moffat \& Vogt (1975) state that No. 7 is a B1 star and this is supported by Lyngå (1977) via his uvby $\beta$ photometry. The listed excesses in the last column of Table 2 have been calculated according to Feinstein \& Marraco (1971), using $U B V$ colors from van den Bergh \& Harris (1976). For No. 4, we have adopted the intrinsic color $(B-V)_{0}=0.91$ of a G5 III star.

\section{Analysis and discussion}

From our polarization measures, 4 objects (members stars 8 and 10 and non-members 17 and 18) have a value for the unit weight error of the fit $\left(\sigma_{1}\right)$ above 1.5. As mentioned before, a higher value gives a clue about the presence of intrinsic polarization in the light from the star. Star member No. 4 with $\sigma_{1}=1.46$ is also a probable member of this group.

Figure 1 displays $P_{\lambda}$ and $\theta_{\lambda}$ vs. $\lambda$ plots for stars with indications of intrinsic polarization. The standard Serkowski's curve (1), the law valid for an interstellar origin in the measured polarization, is also shown. 

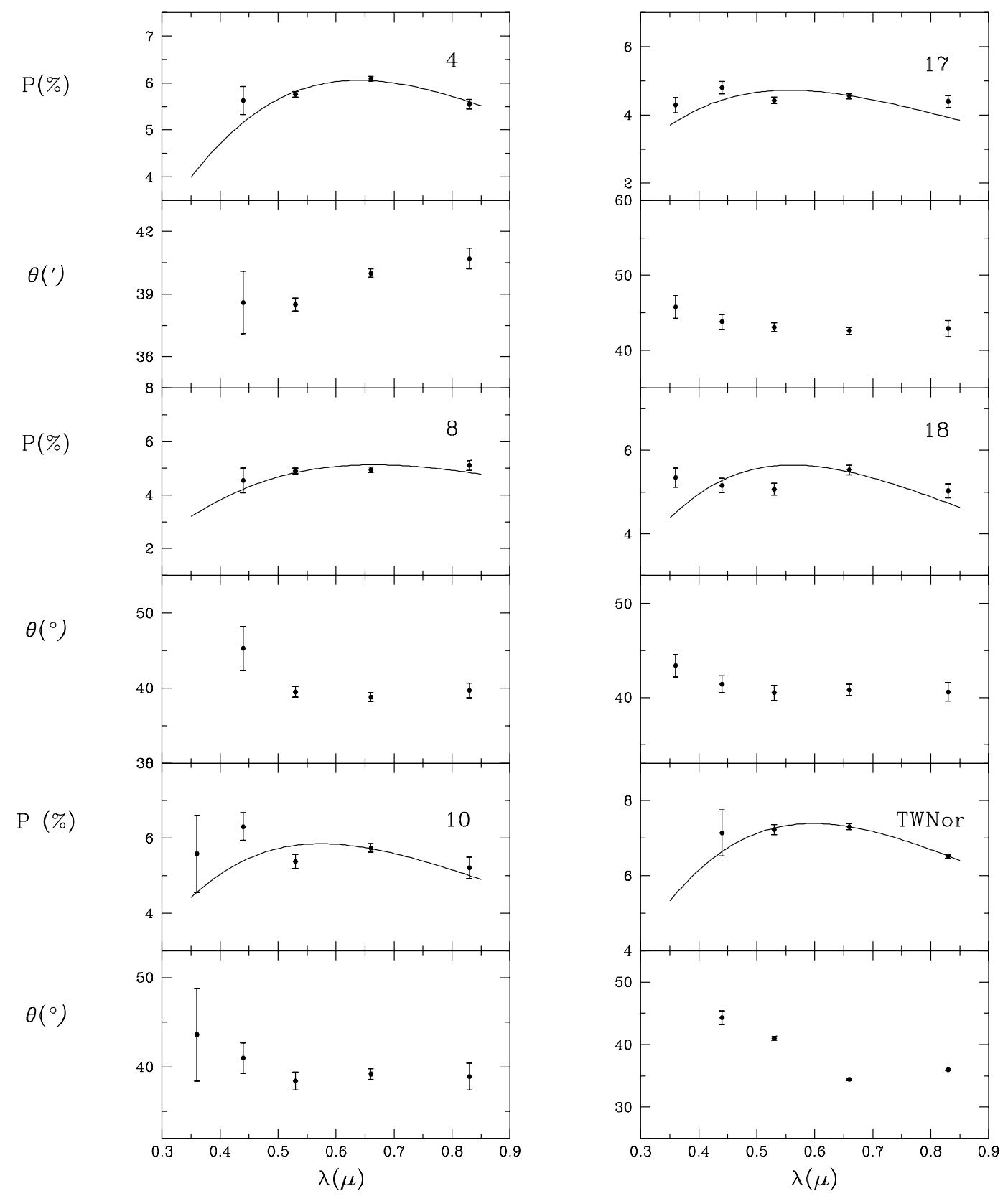

Fig. 1. Intracluster polarization and position angle dependence on wavelength for stars with indications of intrinsic polarization (mismatch between observations and Serkowski's curve fit and/or variable position angle). Plots for the Cepheid TW Nor are also included.

The presence of intrinsic polarization in the light from a star causes a mismatch between observations and the Serkowski's curve fit, and/or a rotation in the position angle of the polarization vector. The abovementioned mismatch is clearly seen only in the plots for stars 10 and 18; but the rotation in the position angle with $\lambda$ is a common characteristic for the whole group of stars with $\sigma_{1}$ higher than 1.5. In the case of stars 17 and 18, the rotation is visible in the $\theta_{U}$ and $\theta_{B}$ values only.

Another indicator of a non-interstellar origin of the measured polarization is a fitted $\lambda_{\max }$ notably smaller when compared to the value $0.55 \mu \mathrm{m}$ for the interstellar medium (Serkowski et al. 1975). But this condition is not fulfilled by any of the stars observed in this investigation.

The ratio $P_{\max } / E_{B-V}$ is known as the "polarization efficiency" of the interstellar medium and it depends mainly on the alignment efficiency and the magnetic field strength. It also depends on the amount of depolarization due to radiation traversing more than one cloud with different field directions. For Lyngå 6 we obtain a mean polarization efficiency of $4.3 \pm 0.3$, close to the value 5.0 found for the ISM (Serkowski et al. 1975). In recent investigations (Orsatti et al. 1998, 2000) we have found values for the efficiency in the direction of several open clusters, 


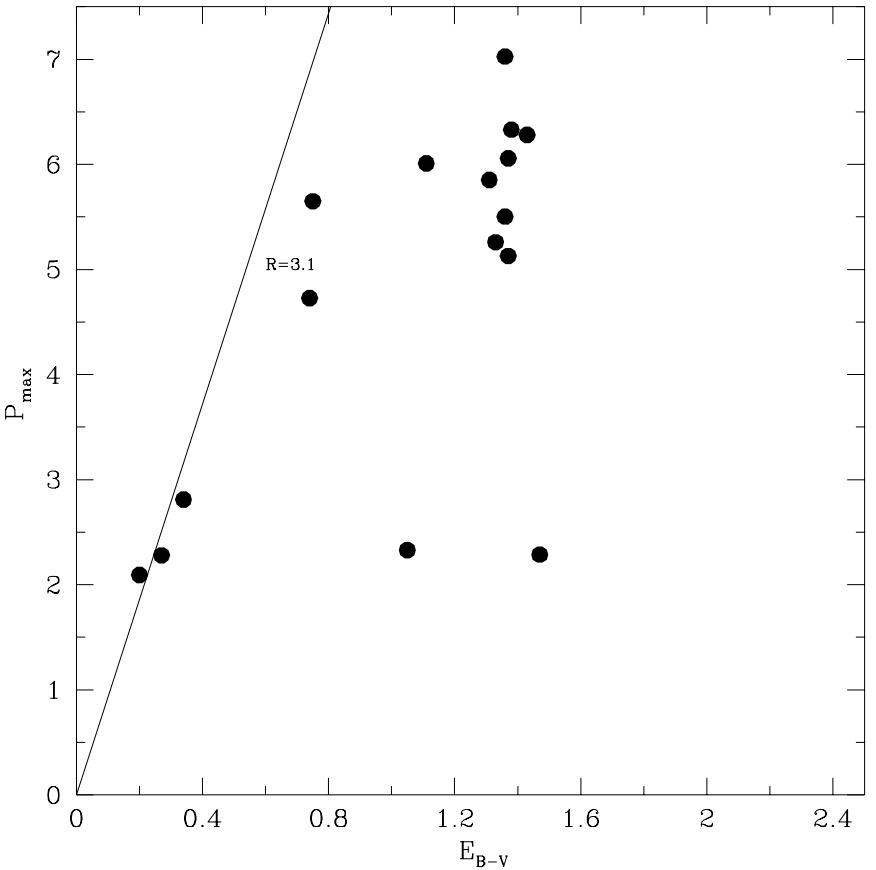

Fig. 2. Polarization efficiency diagrams for the observed parameters. The line of maximum efficiency is drawn using $R_{V}=3.1$.

ranging from 1.28 in Lyngå 14 to 4.2 in NGC 6611. As a consequence, we may say that the polarizing efficiency for the dust in the direction of this cluster is close to the normal value for the interstellar medium.

As can be seen in Fig. 2, where $P_{\max }$ values for the observed stars are plotted versus their $E_{B-V}$ from Table 2, none of the member stars lies to the left of the interstellar maximum line

$P_{\max } \leq 3 A_{v} \simeq 3 R_{v} E_{B-V}$

which is derived for the interstellar dust particles (Hiltner 1956). This situation indicates that the observed polarization is most likely due to the diffuse interstellar material. In the lower part of the plot, non-member stars Nos. 1, 16 and 19 lie very close to the limiting line, in particular the first one, indicating a maximum polarization efficiency. This group of stars have $\overline{P_{\max }}=2.39 \%$ and $\overline{E_{B-V}}=0.27$ mag. According to Neckel \& Klare (1980), the polarization originates in a dust cloud located at about $1 \mathrm{kpc}$ from the Sun in the direction of Lyngå 6, with an $A_{v}$ of about $0.7 \mathrm{mag}$. Stars 14 and 20 (shown in this figure with their excesses $E_{B-V}$ calculated as if they were class $\mathrm{V}$ stars), seem to be evolved stars and very probably part of this group.

The mean polarization for 5 members $(2,4,6,7,9)$ in Lyngå 6, excluding those with $\sigma_{1}$ in excess of 1.5 (Nos. 8 and 10) yields a $\overline{P_{\max }}=5.91 \pm 0.54 \%$ and $\overline{\theta_{V}}=39.4$. The wavelength of maximum polarization for this group amounts to $\overline{\lambda_{\max }}=0.61 \pm 0.03 \mu \mathrm{m}$; that is, dust grains of larger size than those associated with the interstellar medium. These parameters characterize polarimetrically the interstellar material in front of Lyngå 6 .

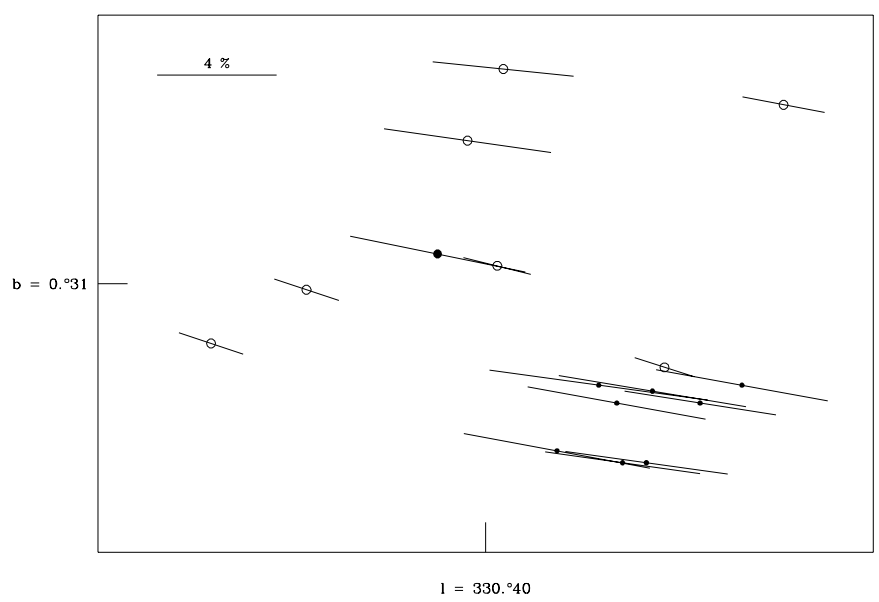

Fig. 3. Observed polarization vectors and their orientations for the observed stars in the direction to Lyngå 6. Open circles are used for non-members and dots for members, according to Lyngå (1977). Star No. 13 is shown with a filled circle. The length of each vector is proportional to the percentage polarization.

In his work, Lyngå (1977) expressed doubts about the membership of stars Nos. 13, 14 and 15 in the cluster. These stars are of the same brightness as the cluster stars but if they were members they would have an excess $E_{B-V}$ smaller by $0.2 \mathrm{mag}$ than the rest. From the polarimetric observation of Nos. 13 and 14 we have arrived at the conclusion that while the first star is a very probable member of Lyngå 6, the second should be a field star. The parameters of the dust in front of No. 13 are $P_{\max }=6.01 \%$ and $\theta_{V}=37.0$, in good agreement with the mean parameters associated with cluster members $\left(5.91 \%\right.$ and $39^{\circ} .4$ respectively). In view of these similarities, we may conclude that this star is a very probable member of the open cluster. Since it is located slightly off the cluster center, the difference in color excess between this star and the rest of the cluster members could be due to differential reddening. For No. 14 the dust parameters through the polarimetric measures are $P_{\max }=2.33 \%$ and $\theta_{V}=34.3$, very different from the ones associated with the open cluster, and this indicates that the star is a foreground object. No. 15 was not polarimetrically observed by us.

Figure 3 plots polarization vectors and their orientations for the observed stars in the direction of Lynga 6 . Open circles are used for non-members and dots for members, according to Lyngå (1977). No. 13 is shown with a filled circle. Figure 4 shows a similar plot, but for stars in the neighborhood of the open cluster, with information from the catalogue of Heiles (2000). As can be seen, field stars and observed members of the cluster both show an alignment of the e-vector along the Sagittarius arm.

TW Nor was first noted as located within the boundaries of the sparse cluster Lyngå 6 by Tsarevskij et al. (1966). Madore (1975) and van den Bergh \& Harris (1976) both were the first to produce evidence for membership of the Cepheid in the cluster. The existence, mean excess and distance to Lyngå 6 have been confidently proved 


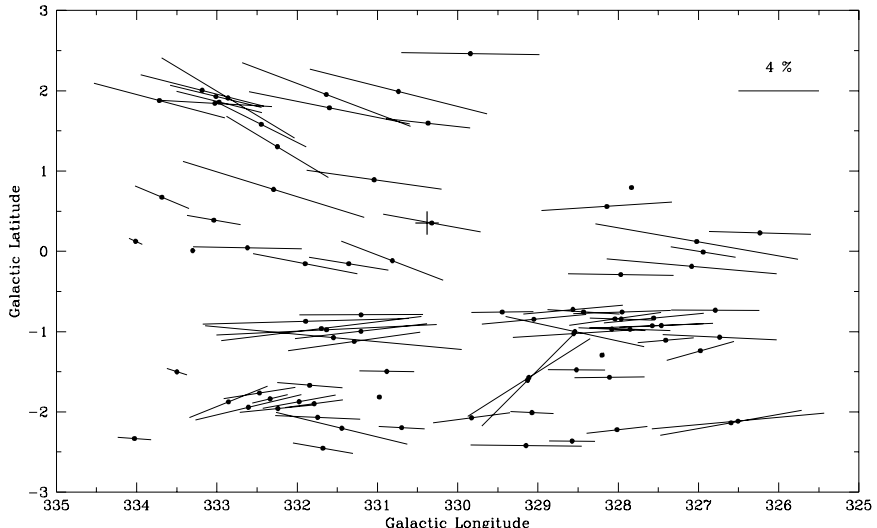

Fig. 4. Polarization vectors and their orientations for stars from Heiles (2000) in the neighborhood of Lyngå 6. The length of each vector is proportional to the percentage polarization. The approximate position of the cluster is indicated with a cross.

by Walker's well-defined main sequence through his color magnitude diagram which reaches $V=20$ mag (Walker 1985), but evidence on the membership of the Cepheid in the cluster Lyngå 6 is not fully conclusive.

The photometric analysis for TW Nor is not fully conclusive concerning its membership in Lyngå 6. Color excesses in the literature range from 1.21 (Laney \& Stobey 1993) to 1.27 mag (Feltz \& McNamara 1980) depending on the different Cepheid's calibrations but some authors adopt for TW Nor the same $E_{B-V}$ as Lyngå 6 (that is, $1.34 \mathrm{mag}$ ). Cepheid parallaxes in the Hipparchos catalogue have only a statistical sense. Concerning proper motion measurements, Hipparcos has measured only the Cepheid but no members of Lyngå 6 . The mean radial velocity of the Cepheid $\left(-56.6 \mathrm{~km} \mathrm{~s}^{-1}\right)$ has been measured by Mermilliod et al. (1987) but we lack equivalent measurements for member stars of Lyngå 6 .

The possibility remains of applying polarimetric techniques to help solve the problem, considering that the polarimetric characteristics of the dust in front of the Cepheid must be compatible with those in front of the cluster. That is, accepting TW Nor as a member of Lyngå 6 means that its excess $E_{B-V}$ should be in line with the mean excess determined for the cluster: $1.34 \pm$ $0.01 \mathrm{mag}$ (Walker 1985). But while the light coming from its neighboring stars (Nos. 2, 4 and 9) shows $P_{\max }$ polarizations of $6.36,6.06$ and $6.33 \%$ respectively, the polarimetric measurements for TW Nor give a higher value for this star: $7.39 \pm 0.05 \%$. The vectorial $\overline{P_{\max }}$ for the cluster is $5.91 \pm 0.54 \%$ and the quoted value for the Cepheid $\left(P_{\max }=7.39 \%\right)$ is outside the $2 \sigma$ interval. If TW Nor were a member of Lyngå 6 , we should find a reason accounting for this excess in polarization in the light coming from the star. Cepheids do not display intrinsic polarization, so we can rule out this kind of explanation. There exists the possibility of having a minute dust cloud located just precisely in the direction of TW Nor. We note that for the closest stars (Nos. 2, 4 and 9) the $E_{B-V}$ reaches the highest value in the open cluster, meaning that there exists a certain concentration of dust toward the central portion of the cluster, where the Cepheid is located. If we suppose a situation of maximum efficiency for the polarizing dust within Lyngå 6, using the relation (2) and taking a value for $R$ of 3.1 , we find that in order to get a percentage of polarization of at least 1.48 (that is the vectorial difference between the $\overline{P_{\max }}$ of the cluster minus the $P_{\max }$ of the Cepheid), the total $E_{B-V}$ in front of the Cepheid must reach at least $1.51 \mathrm{mag}$.

There exists also the possibility of considering the Cepheid as a non-member of the cluster Lyngå 6. In fact this idea has been raised more or less openly by several investigators. For example, Turner (1985) gave a list of 12 Cepheids located within the cluster nuclei boundaries but he omitted TW Nor based upon results of a number of studies which suggested that it may not be a cluster member. In his work, Anderson et al. (1985) stated a discrepancy between the distance moduli of the cluster $\left(V_{0}-M_{V}=11.1 \mathrm{mag}\right)$ and that of the Cepheid as derived from an infrared P-L relation, that is $11.8 \pm 0.2 \mathrm{mag}$ (Welch et al. 1985). Also, works of Fernie \& McGonnegal (1983) and Opolski (1984) put TW Nor at distance moduli of 11.8 and $12.2 \mathrm{mag}$ respectively. Fernie (1990) eliminated TW Nor when deriving his regression formulas. Di Benedetto (1994) gives for the Cepheid a $V_{0}-M_{V}$ of $11.4 \mathrm{mag}$ with a Period-Radius relation and $K$ photometry. More recently, Berdnikov et al. (2000) through $K$-band photometry, placed the object at a distance modulus of $11.6 \mathrm{mag}$ (2080 pc) while the "Database of Galactic classical Cepheids" (Fernie et al. 1995) adopted 11.8 mag (2245 pc) for its distance modulus.

\section{Conclusions}

Star members of the open cluster Lyngå 6 show high polarization values, partly due to the effects of a dust cloud located about $1 \mathrm{kpc}$ from the Sun, in the Local arm. Due to the lack of suitable foreground stars, it was not possible to analyze in this investigation whether there exists dust associated with the cluster itself. The polarizing efficiency for dust in the direction of the cluster is close to the normal value for the ISM. We have found that 2 out of 7 observed member stars show indications of intrinsic polarization.

For different reasons, the currently available membership tests for the Cepheid TW Nor (photometry, radial velocity, parallax and proper motion measurements) have failed in probing its membership of the open cluster. The polarimetric observations, which give information on the characteristics of the dust located just in front of the Cepheid, indicate that the only possibility for TW Nor to be a member of the open cluster relies apparently on the existence of a minute dark cloud located just in front of the object. This situation is possible but highly improbable. The resonable solution is to locate the Cepheid at a certain distance behind the cluster, as previously suggested in other investigations. In that Galactic direction we are 
looking across the Sagittarius arm, so it is reasonable for the dust associated with the arm between the spatial locations of both Lyngå 6 and TW Nor to be responsible for the measured polarization.

To further confirm this result we suggest obtaining polarization measurements of the highly reddened background stars detected by Walker (1985) in his $3^{\prime} \times 2^{\prime}$ field centred on the cluster.

Acknowledgements. We wish to acknowledge the technical support and hospitality at CASLEO during the observing runs. We also aknowledge the use of the Torino Photopolarimeter built at Osservatorio Astronomico di Torino (Italy) and operated under agreement between Complejo Astronomico El Leoncito and Osservatorio Astronomico di Torino. Special thanks go to Carlos Feinstein for valuable comments, and to Mrs. M. C. Fanjul de Correbo and Mr. Ruben Martinez for technical assistance.

\section{References}

Anderson, E. R., Madore, B. F., \& Pedreros, M. H. 1985, in Cepheids: theory and observations, ed. B. F. Madore (Cambridge Univ. Press), IAU Coll., 82, 203

Berdnikov, L. N., Dambis, A. K., \& Vozyakova, O. V. 2000, A\&AS, 143, 211

Caldwell, J. A. R., \& Coulson, I. M. 1987, AJ, 93, 1090

Clocchiati, A., \& Marraco, H. G. 1988, A\&A, 197, L 1

Di Benedetto, G. P. 1994, A\&A, 285, 819

Feinstein, A., \& Marraco, H. G. 1971, PASP, 83, 218

Feltz, K. A. , \& McNamara, D. H. 1980, PASP, 92, 609

Fernie, J. D., Beattie, B., Evans, N. R., \& Seager, S. 1995, IBVS, 4148

Fernie, J. D., \& McGonegal, R. 1983, ApJ, 275, 732

Fernie, J. D. 1990, ApJ, 72, 153
Heiles, C. 2000, AJ, 119, 923

Hiltner, W. A. 1956, ApJS, 2, 389

Knude, J. 1992, A\&AS, 92, 841

Laney, C. D., \& Stobie, R. S. 1993, MNRAS, 263, 921

Lyngå, G. 1964, Lunn Medd. Ser. II, 140

Lyngå, G. 1977, A\&A, 54, 311

Madore, B. F. 1975, A\&A, 38, 471

McMillan, R. S. 1978, ApJ, 225, 880

Mermillod, J. C., Mayor, M., \& Burki, G. 1987, A\&AS, 70, 389

Moffat, A. F. J., \& Vogt, N. 1975, A\&AS, 20, 155

Neckel, T. H., \& Klare, G. 1980, A\&AS, 42, 251

Opolski, A. 1984, Inform. Bull. on Variable Stars, Commission 27, IAU, 2688

Orsatti, A. M., Vega, E. I., \& Marraco, H. G. 1998, AJ, 116, 266

Orsatti, A. M., Vega, E. I., \& Marraco, H. G. 2000, A\&AS, 144,195

Schmidt, E. G. 1983, AJ, 88, 104

Serkoswki, K. 1973, in Interstellar Dust and Related Topics, ed. J. M. Greenberg, \& H. C. van der Hulst (Reidel, DordrechtHolland), IAU Symp., 52, 145

Serkowski, K., Mathewson, D. L., \& Ford, V. L. 1975, ApJ, 196, 261

Thé, P. S. 1977, A\&A, 60, 423

Tsarevskij, G. S., Ureche, V., \& Efremov, Y. N. 1966, Astr. Circ., USSR, 367

Turner, E. G. 1985, in Cepheids: Theory and observations, ed. B. F. Madore (Cambridge Univ. Press), IAU Colloq., 82, 205

van den Bergh, S., \& Harris, G. L. H. 1976, ApJ, 209, 765

Walker, A. R. 1985, MNRAS, 213, 889

Welch, D. L., Wieland, F., McAlary, C. W., et al. 1985, ApJS, 54,547

Wilking, B. A., Lebofsky, M. J., Martin, P. G., Rieke, G. H., \& Kemp, J. C. 1980, ApJ, 235, 905 